\title{
Effect of Cryogenic Treatment on Film-substrate Bonding Strength of DLC Film/High-speed Steel
}

\author{
Yang ZHAO, Chao-Yin NIE*, Yan-Zhong NIE, Xiao-Dong LIU, \\ Xiao-Ling GONG
}

Faculty of Materials and Energy, Southwest University, Chongqing 400715, China

niecy@swu.edu.cn

${ }^{*}$ Corresponding author

Keywords: DLC, Cryogenic Treatment, Film-substrate Bonding Strength, Interface Diffusion, Increase of Grain Boundary.

\begin{abstract}
In order to investigate the effect of cryogenic treatment (CT) on film-substrate bonding strength, $2 \mu \mathrm{m}$ thick DLC film was deposited on quenched W9 high-speed steel by unbalanced magnetron sputtering technique followed by CT at $-196^{\circ} \mathrm{C}$ for 30 hours. Samples before and after CT were characterized respectively by using AFM, indentation, scratch, SEM combined with EDS and XRD analysis. The results show that the film-substrate bonding strength of DLC film/quenched W9 high-speed steel is improved by CT for $30 \mathrm{~h}$. Additionally, the structure of substrate is refined and carbon content in substrate surface of interface significantly increases. Moreover, CT allows the occurrence of martensitic transformation and decomposition increasing substrate grain boundary which facilitate the interface diffusion of carbon from DLC to the substrate. The element $\mathrm{C}$ diffusing to grain boundaries of the substrate surface is proposed to form new carbides which enhance the pinning effect of DLC film on the substrate.
\end{abstract}

\section{Introduction}

Diamond like carbon (DLC) is a kind of amorphous carbon film formed by hybridization of sp3 and sp2 bonds. It is widely used as a novel hard film material in the fields of mechanical, electronic, optical, medical and so on due to the excellent performances [1 2]. However, the drawbacks such as poor fracture toughness and weak bonding strength arising from its excessive residual stress place limitations on the extensive application as mechanical materials of functional film. In this regard, the adhesion of DLC film is now improved popularly through doping the third element, introducing a stress buffer layer or by post-annealing etc. Nevertheless, these methods have their intrinsic limitations [3 4], for example, affecting film purity by introducing intermediate layer or sacrificing film hardness by doping metal element or annealing treatment. Hence, it is of much significance to explore a novel method for the improvement of bonding strength of DLC film-substrate.

Cryogenic treatment (CT), also known as ultra-low temperature processing, can significantly improve the mechanical properties of metal substrate materials such as hardness and wear resistance [5 7]. A lot of studies suggest that CT allows the occurrence of martensitic phase transition and microstructure refinement in high-speed steel alike materials [9 10], but literatures on CT of integrated film-substrate system using this kind of steel materials as substrate had rarely been reported. Our Previous studies have shown that CT doesn't reduce the hardness of DLC film comparing with annealing treatment [8]. However, the features of adhesion of DLC film to the substrate that is a very important property for coating system remains unsolved. It is much worthwhile to make clear how CT will influence bonding strength of DLC film-substrate composite system and whether the mechanism applied to high-speed steel or other alloy steel is still equally applicable to the film-substrate system. The objective of this work is to elaborate the effect of CT on the bonding strength of film-substrate composite system and the mechanism therein using steel as the substrate based on the case study of DLC film/quenched W9Mo3Cr4V high-speed steel system. 


\section{Experimental Materials and Testing Methods}

Quenched W9Mo3Cr4V high-speed steel with chemical composition was selected as substrate material. Magnetron sputtering technique was adopted to deposit DLC films on substrate samples in size of $12 \mathrm{~mm} \times 15 \mathrm{~mm} \times 2 \mathrm{~mm}$ which had been ultrasonically cleaned in acetone and anhydrous ethanol respectively for $10 \mathrm{~min}$. During depositing the film, intermediate transition layers of $\mathrm{Cr}$ and $\mathrm{Cr}_{3} \mathrm{C}_{2}$ were sequentially introduced. By adjusting the deposition time, the total film thickness of all the samples was controlled at about $2 \mu \mathrm{m}$. The preparation process of films was similar to previous research [9]. The liquid method of direct cryogenic in liquid-nitrogen was used in this study. Briefly, samples in the same batch were placed in a liquid nitrogen tank and held for $10 \mathrm{~h}, 20 \mathrm{~h}, 30 \mathrm{~h}$ and $40 \mathrm{~h}$ respectively followed by restoring to room temperature in air. The optimum cryogenic holding time was determined as $30 \mathrm{~h}$ by repeated trials. Empirically, the holding time of $30 \mathrm{~h}$ was adopted in the CT process of this work. The film-substrate bonding strength of DLC film/high-speed steel was qualitatively inspected by using HR-150A typological Rockwell hardness tester. In order to ensure the reliability of test data, test for each sample was repeated for three times under the same conditions. Nanoscratch tests were respectively conducted to measure the film-substrate bonding force of DLC film/high-speed steel within a fixed area of the same sample. The sectional profile of main elements for the coated samples was scanned by energy dispersive spectroscopy (EDS). Prior to measurement, the coated samples were inlaid in phenolic resin and the sections were ground and polished well. The metallurgy microstructure was observed by virtue of scanning electron microscopy (SEM) and the phase structure of high-speed steel substrate was analyzed by using X-ray diffractometer (XRD).

\section{Results and Discussion}

\section{Film-substrate Bonding Strength}

Film-substrate bonding strength of DLC coating system before and after CT is qualitatively assessed in accordance with the damage made by Rockwell indentation, and the SEM morphologies of the indentation imprints are shown in Fig.1. The observed white matter attaching to the periphery of indentation imprint (as the white arrow indicated) is considered as the shedding debris of DLC film generated during indentation for both specimens before and after CT. In the process of unloading, due to the differences in elastic-plastic properties between DLC film and the substrate, greater elastic-plastic mismatch and larger residual stress existing between DLC film and substrate may lead to the emergence of ring-like cracks in DLC film. As shown in Fig.1 (a), the continuous and comparative inerratic shedding of DLC film occurs along the entire indentation circumference while the film in nearly three-quarters of cavity side of indentation is creased (the black arrow). Nevertheless, in comparison, the shedding of DLC film after CT along indentation circumference shown in Fig.1 (b) becomes intermittent and significantly reduced with the creased film being much decreased (as the black arrow indicated).
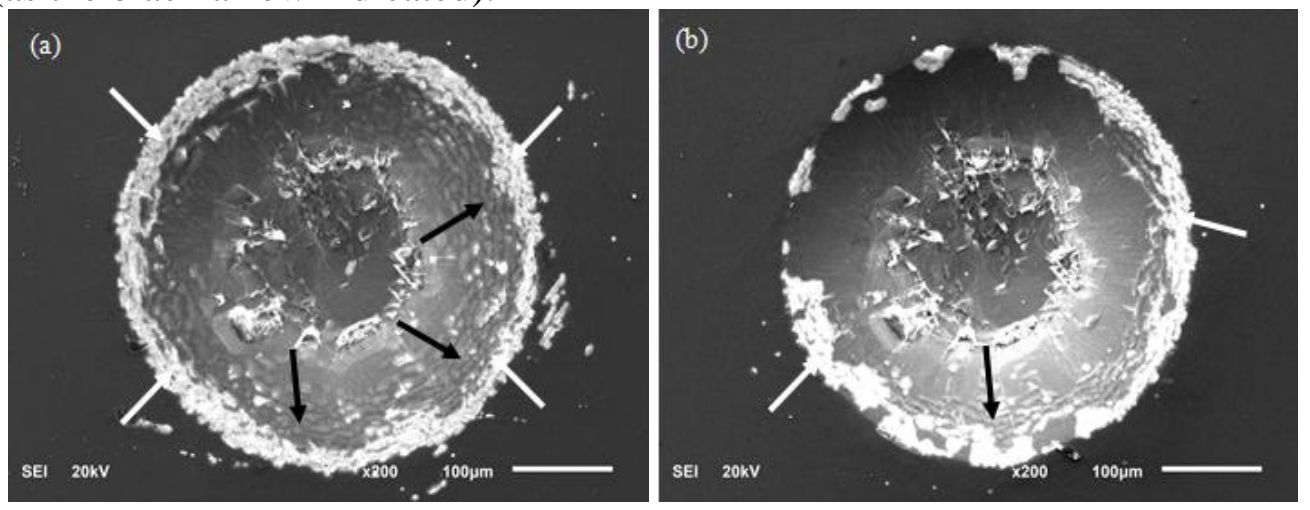

Fig.1 SEM morphology of Rockwell indentation of DLC film/high speed steel (a) Before CT; (b) After CT. 
Nanoscratch test is carried out to measure film-substrate bonding force of the studied coating system before and after CT. The film-substrate bonding strength is evaluated in terms of critical load which is defined as the onset force when the film failure occurs [10]. The scratch and loading curves as function of displacement of indenter are displayed in Fig.2, and the corresponding scratch image recording the practical status of the film is placed above the plots as an inset. Because the elastoplastic deformation ability of high-speed steel substrate is stronger than that of DLC film, the sharp change in scratch depth in case of a failure of the film may be reflected on scratch curve as an obvious inflection point which is progressively confirmed by the attached image, and the critical load corresponding to the inflection point can be read from the loading curve. By this methodology, the film-substrate bonding forces of DLC film/high-speed steel before and after CT are about $1.1 \mathrm{~N}$ and $1.5 \mathrm{~N}$ respectively, which is in line with the result of Rockwell indentation method.
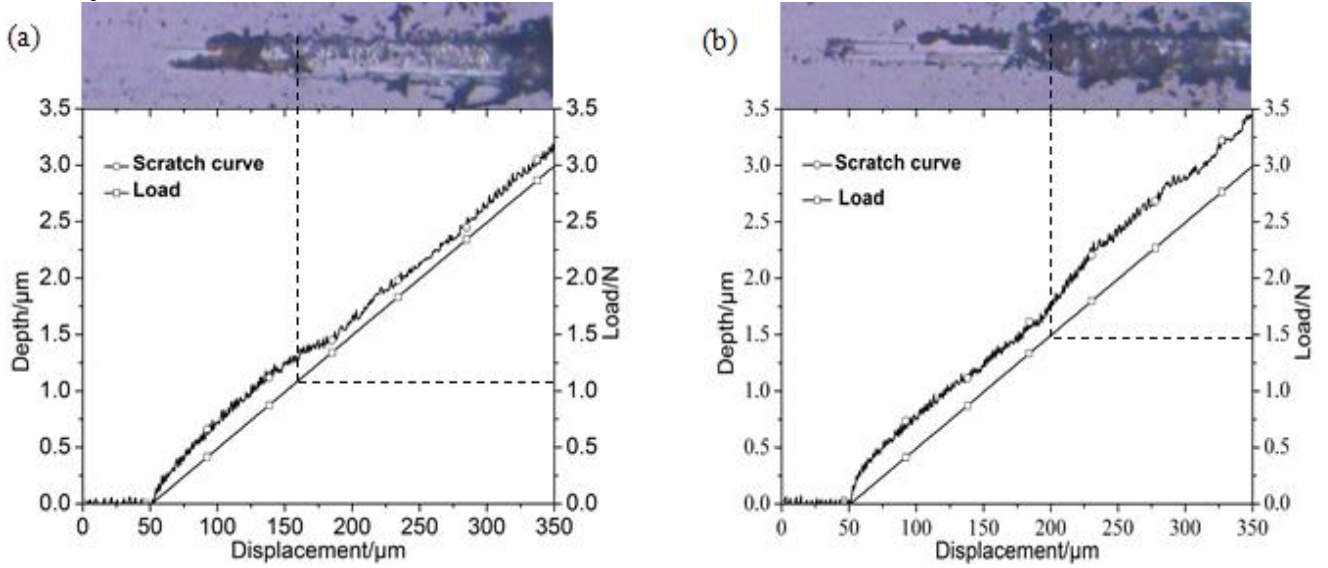

Fig.2 Schematic illustration for determination of bonding force of DLC film/high speed steel (a) Before CT; (b) After CT.

\section{Compositional Profile of Interface}

The cross-sectional SEM morphology and line-scanning EDS compositional profiles of DLC film/high-speed steel before and after CT are exhibited in Fig.3. The SEM morphology reveals the thickness of DLC layer and the couple intermediate layers $\left(\mathrm{Cr}_{3} \mathrm{C}_{2}, \mathrm{Cr}\right)$ are about $1 \mu \mathrm{m}, 0.5 \mu \mathrm{m}$ and $0.5 \mu \mathrm{m}$ respectively. Though the film-substrate discrepancy in physical properties may result in unstable interfacial microstructure, the volume shrinkage under the condition of CT tends to make the interfacial microstructure denser, enhancing the coalescence of defects such as voids and pores and thus inducing the concentration change of certain elements at the interface.

It should be noted that the EDS curves for cryogenic treated sample exhibit lower intensity due to the shorter line-scanning time, whereas the elaboration on compositional distribution of samples before and after CT will not be affected. It can be seen from the line-scanning profiles in Fig. 3 that the content distributions of element $\mathrm{C}$ for film layers before and after $\mathrm{CT}$ present gradient distribution that begin with an increasing range followed by gradual decreasing to constant value at the interface (reaching a thickness of $2.0 \mu \mathrm{m}$ ). Mutual diffusion of various elements occurred between adjacent layers or between film and substrate in the process of physical vapor deposition. As a result, the $\mathrm{C}$ atoms with higher bombardment energy could diffuse through the film especially when DLC is deposited on intermediate layer $\left(\mathrm{Cr}_{3} \mathrm{C}_{2}\right)$, and a portion of $\mathrm{C}$ atoms even sequentially penetrate structural clearance of intermediate layers $\left(\mathrm{Cr}_{3} \mathrm{C}_{2}, \mathrm{Cr}\right)$ to substrate.

Furthermore, comparing line-scanning curves in Fig.3 reveals a reduced distribution gradient of element $\mathrm{C}$ for film/substrate undergoing $\mathrm{CT}$. The relative content of carbon in the range of $0-1.0 \mu \mathrm{m}$ of DLC layer is significantly decreased on account of CT implying lowered actual content of element $\mathrm{C}$ in DLC layer. It should be noted that element $\mathrm{C}$ distributes in $\mathrm{Cr}$ layer located at depth of $1.5 \sim 2.0 \mu \mathrm{m}$ at a mitigated gradient, and in return its average relative content throughout the film to $2.0 \sim 3.0 \mu \mathrm{m}$ depth of substrate increases. These behaviors suggest that a portion of atomic $\mathrm{C}$ may penetrate the structural clearance of $\mathrm{Cr}_{3} \mathrm{C}_{2}$ layer and diffuse to $\mathrm{Cr}$ layer and the substrate surface when it was 
brought to room temperature following CT insulation. It can be explained that CT can evidently modify the microstructure of high-speed steel and consequently facilitates the diffusion of atomic $\mathrm{C}$ from DLC film to the substrate.
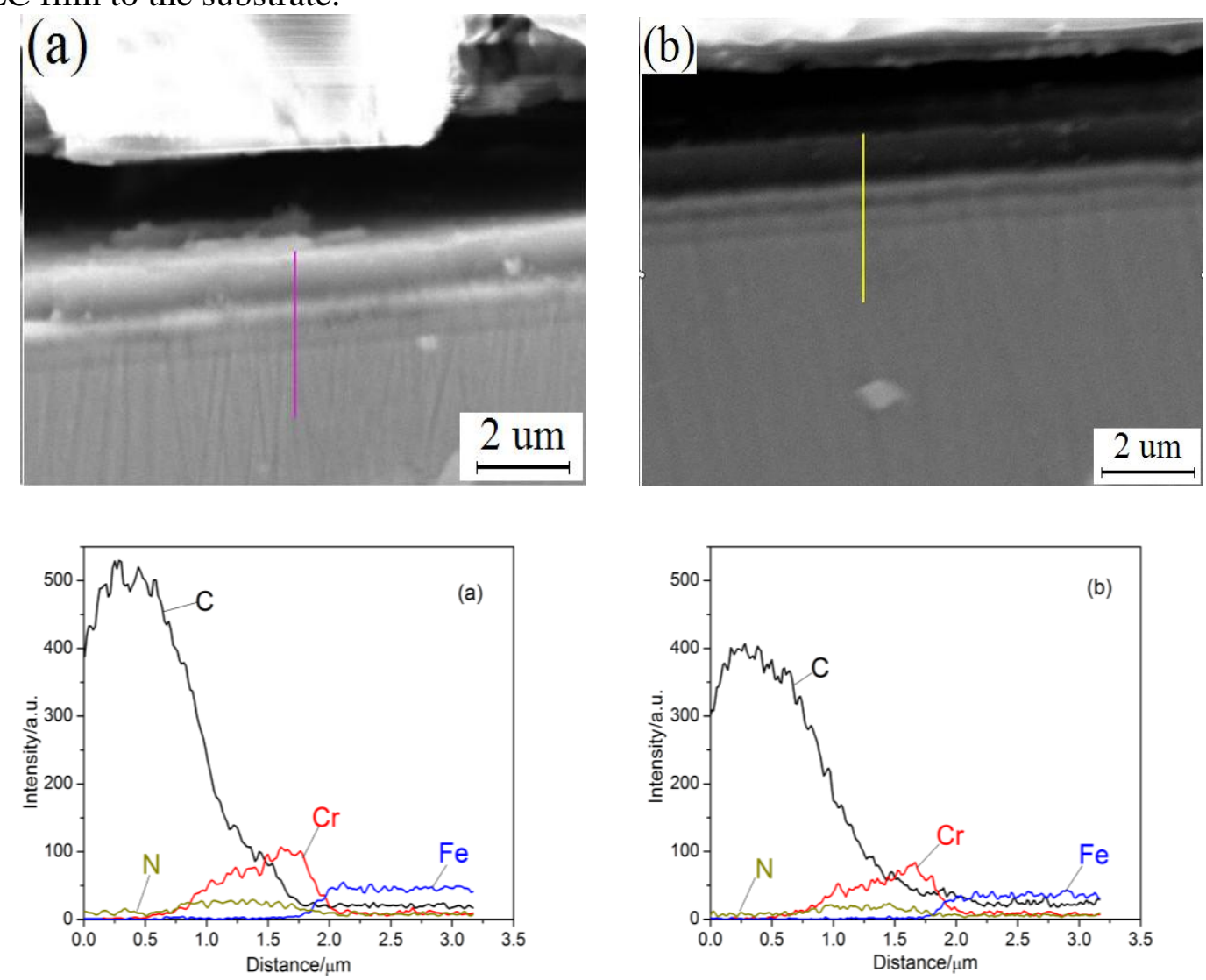

Fig.3 Cross-sectional SEM morphology and compositional profiles of DLC film/high speed steel (a) Before CT; (b) After CT.

\section{Morphology of Substrate}

The SEM images of surface morphology of quenched high-speed steel before and after CT are shown in Fig.4. It can be seen from Fig.4 that fewer black plate martensites but relatively more white residual austenites in clumps distributed unevenly in matrix of the steel before CT. After CT, black plate-like martensites increase and uniformly distribute, and instead the white residual austenites in clumps decrease to some extent. Fig.4 (b) also shows that the comparatively wide area of dislocation group column exists around the plate incomplete twin martensite obtained in the process of CT [11], equivalent to enlarging dislocation substructure area and the further refinement of martensite texture. Meanwhile, the substructures of almost isometric clump-like residual austenites retained during CT are constructed of dislocation and twin crystals breaking into more blocky units internally.
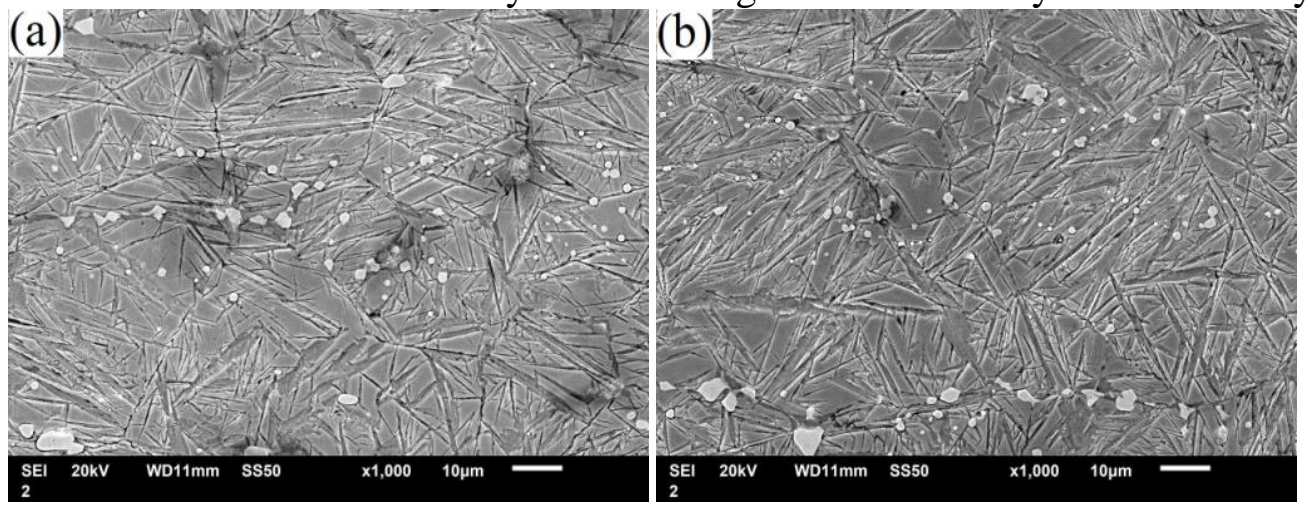

Fig.4 SEM surface morphology of high-speed steel substrate (a) Before CT; (b) After CT. 
The XRD patterns of quenched high-speed steel before and after CT shown in Fig.5 can further verify the transformation of residual austenite to martensite through CT. Relative contents of residual austenite, martensite and carbide of substrate before and after CT are determined by calculating the areas of corresponding diffraction peaks using datum-line based integration method. The computed results are summarized in table 1 , indicating that the relative content of residual austenite decreases while that of martensite increase apparently. Because the solid solubility of carbon in martensite declines at the insulation stage of CT process, a large number of fresh ultrafine dispersive carbide particles precipitated along martensite twin band and dislocation line leading to the decomposition and refinement of martensite structure [12]. The increasing grain boundaries of substrate induced by martensitic phase transition and decomposition in the process of CT will provide more downward channels for diffusion of carbon atoms through film to substrate as well as new locations for the intergranular segregation. In general, the intergranular segregated carbon atoms in high alloy steel can combine with other alloying elements of grain boundary to form various carbides [13]. Therefore part of $\mathrm{C}$ atoms from film layer seem to form carbides in boundaries of substrate surface enhance pinning effect of DLC film on substrate and hence favors the improvement of interfacial bonding strength.

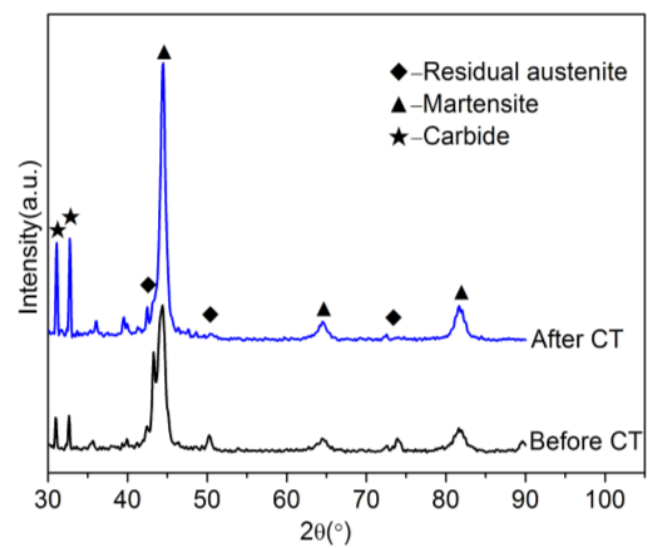

Fig.5 XRD patterns of high-speed steel substrate (a) Before CT; (b) After CT.

Tab. 1 The phase's relative percentage content of the high speed steel substrates

\begin{tabular}{cccc}
\hline Processing method & Residual austenite(\%) & Martensite(\%) & Carbide(\%) \\
\hline Untreated & 28.7 & 65.0 & 6.3 \\
Cryogenic treated & 12.4 & 80.0 & 7.6 \\
\hline
\end{tabular}

Generally, the required activation energy of grain boundary diffusion is less than that of bulk diffusion in diffusion mechanism, because the grain boundary containing a lot of metallurgy flaws is characteristic of serious lattice distortion greatly increasing atomic energy that allows for a higher frequency of atom leap in favor of materials diffusion. Hence, only the grain boundary diffusion can occur at lower temperature and is the dominant diffusion mechanism in the process of CT. The more the volume fraction of grain boundaries, the more the "short-range diffusion channel" composed of grain boundary. Increase in grain boundary of high-speed steel owing to CT will give rise to the short-range channels for downward diffusion of elements from film layer, especially for the grain boundary of high-energy non-equilibrium twin martensite generated by martensitic transformation. $\mathrm{CT}$ is beneficial for the downward diffusion of element $\mathrm{C}$ in film layer enhancing the interfacial pinning effect of film-substrate, and eventually improving film-substrate bonding strength.

\section{Summary}

(1) The film-substrate bonding force of DLC film/quenched W9 high-speed steel is improved by cryogenic treatment for $30 \mathrm{~h}$. Additionally, the structure of substrate is refined and carbon content in the substrate surface of film-substrate interface significantly increases. 
(2) Cryogenic treatment allows the occurrence of martensitic transformation and decomposition increasing substrate grain boundary which facilitate the diffusion of carbon from DLC film to the substrate.

(3) The element $\mathrm{C}$ diffusing to grain boundaries of the substrate surface are proposed to form new carbides which enhance the pinning effect of DLC film on the substrate, eventually improving the film-substrate bonding strength of DLC film/quenched W9 high-speed steel.

\section{Acknowledgements}

The author would like to thank support of National Natural Science Fund of China (NSFC, Grant No.51271153).

\section{References}

[1] C.R. Lina, D.H. Wei and C.K. Chang: Phys. Procedia Vol. 18 (2011), p. 46.

[2] C.A. Charitidis: Int. J. Refract. Met. Hard Mater Vol. 28 (2010) No.1, p. 51.

[3] H.F. Liang, Z.H. Liang, C.L. Liu and L.G. Meng: Appl. Surf. Sci Vol. 256 (2010), p. 1951.

[4] Y.H. Lin, H.D. Lin, C.K. Liu: Thin Solid Films Vol. 518 (2009), p. 1503.

[5] A. Eari: ASM Handbook Vol. 4 (1991), p. 203.

[6] D.N. Collins: Heat Treat. Met Vol. 24 (1997) No.3, p. 71.

[7] K.T. Luo, P.W. Kao and D. Gan: Metal Mater. Eng Vol. 151 (1992), p.15.

[8] X. Li and S.Y. Li: J. Shanghai Jiaotong. Univ Vol. 36 (2003) No.7, p. 905. (In Chinese)

[9] C.Y. Nie, B.Y. Zhang and H.M. Xie: Acta Metall. Sinica Vol. 43 (2007) No.11, p. 1207. (In Chinese)

[10] G. Wei, T.W. Scharf and J.N. Zhou: Surf. Coat. Technol Vol. 357 (2001), p. 146.

[11] D.N. Collins: Heat Treat. Met Vol. 23 (1996) No.2, p. 40.

[12] G. Chen, Q.S. Wang, J.C. Han and M.Z. Zhou: J. Hunan. Univ.(Nat. Sci.) Vol. 39 (2012) No.4, p. 53. (In Chinese)

[13] M.Y. Zhang, K. Yang: Rare Metal Mater. Eng Vol. 42 (2013) No.7, p. 1531. (In Chinese) 